associates, in some children treated with TPM should not appreciably alter the anticonvulsant effect.

\title{
CYTOKINES AND FEBRILE CONVULSIONS
}

The production of pro-inflammatory cytokines (IL-1B, IL-6, and TNF-a), and anti-inflammatory cytokine IL-10 by peripheral blood mononuclear cells (PBMC) from 13 children 2 weeks after a febrile convulsion and in 11 without a history of febrile convulsion, was studied at the C Schneider Children's Medical Center, Rabin Medical Center, and Sackler School of Medicine, Tel Aviv University, Israel. Both groups showed an increase in all types of cytokine production, but the secretion of IL-6 and IL-10 in response to lipopolysaccharide stimulation of PBMC was greater in those with a history of febrile convulsions. (Straussberg R, Amir J, Harel L et al. Pro- and anti-inflammatory cytokines in children with febrile convulsions. Pediatr Neurol 2001;24:49-53). (Respond: Dr Bessler PhD, Laboratory for Immunology and Hematology, Rabin Medical Center, Golda Campus, Petah Tiqva, Israel).

COMMENT. Cytokine production by PBMCs of children with a history of febrile seizures at steady state differs from those without convulsions. Infection stimulates immune cells to produce pro-inflammatory cytokines, that can affect brain function and result in seizures. IL-10 inhibits the production of proinflammatory cytokines and has an antipyretic effect. The increased production of IL-10 in the above patients with seizures may be a defense mechanism. The authors comment that cytokine profiles of patients with febrile convulsions and their families may be worthwhile.

\section{HEADACHE DISORDERS}

\section{ACUTE HEADACHE AS AN EMERGENCY PRESENTATION}

A consecutive series of 150 children (aged 2 to 18 years) who presented to an emergency department with abrupt onset of severe headache were evaluated prospectively at the Children's Hospital of the King's Daughters, East Virginia Medical School, Norfork. Causes of acute headache included upper respiratory tract infection with fever (57\%), migraine (18\%), viral meningitis $(9 \%)$, posterior fossa tumor $(2.6 \%)$, ventriculoperitoneal shunt malfunction (2\%), epileptic seizure with postictal headache $(1.3 \%)$, concussion $(1.3 \%)$, intracranial hemorrhage $(1.3 \%)$, and undetermined $(7 \%)$. The URI was viral in $39 \%$, sinusitis in $9 \%$, and streptococcal in $9 \%$. Occipital location and an inability to describe the quality of pain were symptoms significantly associated with serious underlying causes. Pain intensity was not a discriminating factor. Normal activity levels were indicative of a URI-related cause and against serious pathology. Patients with migraine, URI, post-traumatic, and postictal headache had normal neurologic exams. Patients with brain tumor or hemorrhage had obvious neurologic signs, including ataxia, hemiparesis, abnormal eye movements, and papilledema. (Lewis DW, Oureshi F. Acute headache in children and adolescents presenting to the emergency department. Headache March 2001;40:200-203). (Respond: Dr Donald W Lewis, Child and Adolescent Neurology, 850 Southampton Avenue, Norfolk, VA 23510).

COMMENT. The most common cause of abrupt onset of severe headache in children is an upper respiratory tract infection with fever, sinusitis, or migraine. These children may maintain normal activities, the headache is not occipital in location, and the neurologic exam is normal. An occipital headache associated with abnormal neurologic signs is indicative of a serious structural pathology. 